\title{
Engineering a Localized Surface Plasmon Resonance Platform for Molecular Biosensing
}

\author{
Sajid Farooq, Renato E. de Araujo* \\ Laboratory of Biomedical Optics and Imaging, Federal University of Pernambuco, Recife, Brazil \\ Email: *renato.earaujo@ufpe.br
}

How to cite this paper: Farooq, S. and de Araujo, R.E. (2018) Engineering a Localized Surface Plasmon Resonance Platform for Molecular Biosensing. Open Journal of Applied Sciences, 8, 126-139. https://doi.org/10.4236/ojapps.2018.83010

Received: January 27, 2018

Accepted: March 19, 2018

Published: March 22, 2018

Copyright ( $) 2018$ by authors and Scientific Research Publishing Inc. This work is licensed under the Creative Commons Attribution International License (CC BY 4.0).

http://creativecommons.org/licenses/by/4.0/

(c) (i) Open Access

\begin{abstract}
In this work, we introduce a new perspective on the development of Localized Surface Plasmon Resonance (LSPR) optical biosensors. Computational simulations, focused on the assessment of the LSPR spectrum and spatial distribution of the electromagnetic field enhancement near a metallic nanoparticle, elucidated the behavior of crucial parameters, as figure of merit, bulk and molecular sensitivity, which governs a LSPR sensor performance. Gold and silver nanospheres were explored as starting point to assess plasmonic optical characteristics of the nanostructured sensor platform. Here, for the first time in the literature, Campbell's model was evaluated exploiting a NP size-dependence approach. The theoretical analyses indicate a nonlinear behavior of the bulk and molecular sensitivity as function of the NP size. Substantial LSPR peak shifts due to the adsorption of molecules layer on a NP surface were observed for nanoparticles with $\sim 5 \mathrm{~nm}$ and $\sim 40 \mathrm{~nm}$ radius. Moreover, on molecular sensing, LSPR peak shift is also determined by the thickness of adsorbed molecular shell layers. We observed that for $40 \mathrm{~nm}$ radius gold and silver nanospheres, significant LSPR peak shift could be induced by small (few $\mathrm{nm}$ ) thickness change of the adsorbate shell layer. Moreover, this work provides insights on the LSPR behavior due to adsorption of molecular layer on a NP surface, establishing a new paradigm on engineering LSPR biosensor. Furthermore, the proposed approach can be extended to engineer an efficiently use of different nanostructures on molecular sensing.
\end{abstract}

\section{Keywords}

Localized Surface Plasmon Resonance, Optical Sensor, Nanomaterials

\section{Introduction}

The noble metal nanostructures strongly interact with light, resulting in size de- 
pendent absorption, scattering and robust local electric field enhancement. These extraordinary optical characteristics of small particles have led to remarkable interest into their potential applications as nanoscale elements in diverse range of technologies. These optical characteristics are governed due to collective coherent oscillations of free electrons, known as localized surface plasmon resonance (LSPR) [1]. The LSPR spectrum is tunable and reliant on shape, size, composition and on the surrounding dielectric medium [2]. The spectrum of LSPR excitations have been the subject of intensive research efforts and encourage researchers to synthesize the growth of complex shapes nanostructures such as nanoshells [3], nanorice [4], nanocages [5], nanostars [6], nanorods [7] and nanopyramids [8], which shows plasmon peaks in various spectral regions. In general, the shaper tips particles are expect to have LSPR at longer wavelength as compared to spheroids [9]. However, spherical nanostructures provide a starting point to evaluate plasmonic optical characteristics of metals.

Moreover, the size of a metallic nanostructure plays an important rule on the light-NP interaction. In fact, scattering and absorption cross sections are size dependent [10]. On a Quasi Static approximation, absorption cross section of a spherical nanoparticle is proportional to $r^{3}$, while scattering cross section is proportional to $r^{6}$, where $r$ is the radius of nanoparticle. In general, absorption processes will be more effective than scattering on particles with dimension smaller than $10 \mathrm{~nm}$. For larger metallic structures the light-NP interaction will be determined mainly by scattering [11].

In principal, LSPR can be achieved in any metal, semiconductor or alloy by fulfilling the Frohlich condition: $\varepsilon_{r}=-2 \varepsilon_{m}$ [12], where $\varepsilon_{r}$ is the real part of the nanoparticle complex dielectric function and $\varepsilon_{m}$ is permittivity of the NP surrounding non-absorbing medium. Therefore, to establish LSPR on a NP, $\varepsilon_{r}$ should be negative while the imaginary part of the dielectric function, $\varepsilon_{p}$ is expected to be positive and negligible. Gold and silver are the mostly used metals, with negative real and small positive imaginary dielectric values, on LSPR applications [13].

Therefore, gold and silver nanoparticles are increasingly receiving attention as an important starting point for label-free sensing. LSPR sensors can be explored as fast, reliable, low-cost, and fairly simple tool for medical diagnosis. Various examples of LSPR biosensors were attributed to the diagnosis of relevant medical disease, as Alzheimer [14], preeclampsia [15], influenza [16], HIV-1 [17], hepatitis B infections [18] and Dengue virus [19], as well as for intracellular protein sensing [20].

LSPR sensors are mainly based on the identification of plasmon resonance wavelength shift. LSPR peak shift may be induced by changes of the NP surrounding medium or by molecular attachment to the nanostructure surface. On molecular LSPR sensors, when a specific analyte attaches to the surface of NP, a shift of LSPR spectra may be observed. In particular, chemisorption process can 
lead to Self-Assembled Monolayers (SAM) formation on the metallic nanoparticles surface [21]. Moreover several important biomolecules, as antibodies, enzymes, DNAs and proteins, have been associated to metallic nanostructures, providing a diversity of LSPR platform for biomedical applications [1].

To develop an efficient LSPR molecular sensor, an evaluation of plasmon peak shift as a function of the NP size and adsorbate molecule layer thickness is required. Whereas several good reviews on LSPR sensors can be found in the literature [22]-[28]. In this work, crucial parameters that rule the LSPR molecular sensor performance, as figure of merit, bulk sensibility and electromagnetic field distribution around the NP and their dependence with nanostructure size and material composition (gold and silver) are evaluated. Moreover, this work provides insights on the LSPR behavior due to adsorption of molecular layer on a NP surface. Here, for the first time in the literature, Campbell's model is evaluated exploiting a NP size-dependence approach, establishing a new paradigm on engineering LSPR biosensor.

\section{Principals of LSPR Biosensing}

The LSPR wavelength shift $(\Delta \lambda)$ due to a molecular monolayer adsorption on a metallic NP surface can be mathematically described by Campbell's model as [29]:

$$
\Delta \lambda=\eta_{b} \cdot \Delta n \cdot\left(1-\mathrm{e}^{-2 \mathrm{~d} / l_{d}}\right),
$$

where $\Delta n$ is the change in refractive index (RI) due to adsorption layer $\left(\Delta n=n_{\text {ads }}\right.$ - $n_{m}$ where $n_{a d s}$ and $n_{m}$ are the RI of the adsorbate layer and of the surrounding dielectric medium, respectively). Additionally, $l_{d}$ is the EM field decay length around the NP, $d$ is adsorbate layer thickness and $\eta_{b}$ is known as the LSPR bulk sensitivity. Moreover, bulk sensitivity is termed as the variation of LSPR peak position with respect to the change in RI unit (RIU) of the medium, and its unit is eV/RIU or nm/RIU [22]. Thus, bulk sensitivity can be written as [23]:

$$
\eta_{b}=\frac{\Delta \lambda_{\mathrm{LSPR}}}{\Delta n_{d}}
$$

where $\Delta \lambda_{\mathrm{LSPR}}$ and $\Delta n_{d}$ are, respectively, the wavelength shift of LSPR peak and the refractive index change of a medium. Moreover, sensing performance can also be evaluated by exploring Figure of Merit (FoM) factor. The FoM is defined as the ratio of bulk sensitivity to the full width at half maximum (FWHM) and is expressed as [24]:

$$
F O M=\frac{\eta_{b}}{F W H M} .
$$

For nanosphere LSPR-based sensors, the bulk sensitivity, FoM and EM field decay length are reliant on the NP particle radius [1]. Therefore, the plasmon peak shift of LSPR molecular sensor should also be dependent of the nanoparticle size. 


\section{Methods}

\subsection{Mie Theory}

Gustav Mie, in 1908, developed an analytical solution for plane EM wave incident on spherical conducting nanoparticles. From the solution obtained after solving Maxwell's equations, the cross sections of scattering $\left(C_{s c a}\right)$ and absorption $\left(C_{a b s}\right)$ of the small NPs can be described [1]. From $C_{s c a}$ and $C_{a b s}$ the extinction cross section can be obtained as $C_{e x t}=C_{a b s}+C_{s c a}$. We employ the optical efficiency $(Q)$ here instead of cross section $(C)$ for extinction, therefore, $Q$ is defined as the ratio of the optical cross section to the geometric cross section [11].

\subsection{Simulations Analyses}

Computational simulations were used to render the interaction of light with the nanoparticle, and therefore, leading to the understanding of the behavior of crucial parameters that rule the LSPR molecular sensor performance. Here, Finite Element Method (FEM) was performed using COMSOL multiphysics, to determine the frequency domain scattered field distributions and, therefore, the NP extinction cross sections. In FEM simulation, the nanoparticle surface was divided into small tetrahedral mesh elements with 'finer' size. Additionally, anisotropic perfectly matched layer (PML) around the nanoparticle, was introduced to avoid any reflection artifacts on the simulation. The amplitude of the background-oscillating field was set to $1 \mathrm{~V} \cdot \mathrm{m}^{-1}$. The used bulk values of the metal complex (real and imaginary) dielectric functions for $\mathrm{Au}$ and $\mathrm{Ag}$ were obtained from literature [13].

\subsection{Experimental Analysis}

Silver and gold nanospheres colloidal samples with polyvinylpyrrolidone (PVP) stabilizer were obtained from Sigma alderich and Nanocomposix respectively. The samples transmission and emission spectrum, from 300-1100 nm, were obtained using an Ocean Optics spectrophotometer $(\mathrm{HR}+4000)$, with a $1 \mathrm{~nm}$ resolution. For the spectroscopic analyses, the samples were placed in quartz cuvettes of $1 \mathrm{~cm}$ width. Fiber bundles were used to guide light from a Halogen-Deuterium light source to the sample and to send light from sample to the spectrophotometer.

\section{Results and Discussion}

The size of nanoparticle is essential parameter to forecast the performance of a nanostructure biosensor optical platform. Moreover, dielectric function of a metal is also influenced by its structure (size). As the metallic nanoparticle becomes smaller, electron scattering on the NP surface becomes more pronounce, and therefore affecting the dielectric function value of the material. The metallic nanoparticle complex dielectric functions $\left(\varepsilon(\omega)=\varepsilon_{r}+\varepsilon_{i}\right)$ can be described by Drude model, which also account for the conduction electrons scattered on the 
metallic surface. Therefore dielectric function can be written as [30]:

$$
\varepsilon(\omega)=\varepsilon_{\text {inter }}(\omega)+\frac{\omega_{p}^{2}}{\omega(\omega+i \gamma)}
$$

where $\varepsilon_{\text {inter }}(\omega)$ depicts interband transitions, $\omega_{p}$ represents plasmon frequency and $\gamma$ is a phenomenological scattering parameter. For nanoparticles, the scattering parameter has inherent contributions of the intrinsic properties of the material as well as from interface scattering, and therefore, it can be described as $\gamma=\gamma_{b u l k}+\gamma_{s c a t}$ Interface scattering becomes significant when the effective electron path length $L_{\text {eff }}$ is larger than to the nanoparticle itself [31]. The effective path length for convex shapes particles, as sphere, rods, cubes etc, is expressed as $L_{\text {eff }}=4 V / S$ where $V$ is the volume and $S$ is the particle surface area [30]. Thus the scattering parameter is given by $\gamma_{\text {scat }}=A V_{f} / L_{\text {eff }}$ where $A$ is dimensionless parameter, also known as scattering efficiency, usually taken as unity and $V_{f}$ is the Fermi velocity [32]. For gold, $\gamma_{\text {bulk }}=1.07 \times 10^{14} \mathrm{~s}^{-1}, V_{f}=1.40 \times 10^{6} \mathrm{~ms}^{-1}$, while for silver $\gamma_{\text {bulk }}=3.22 \pm 1.22 \times 10^{13} \mathrm{~s}^{-1}$ and $V_{f}=1.39 \times 10^{6} \mathrm{~ms}^{-1}$ [13]. The Figure 1 depicts the dielectric function of $\mathrm{Au}(\mathrm{a}, \mathrm{c})$ and $\mathrm{Ag}(\mathrm{b}, \mathrm{d})$ particles, considering size correction, showing that decreasing the nanoparticle radius (effective path length) both real and imaginary parts of permittivity changes. The real part $\left(\varepsilon_{r}\right)$ depicts the pattern of electron polarization in the medium as a consequence of incident field and determines LSPR peak position. However, imaginary part $\left(\varepsilon_{i}\right)$

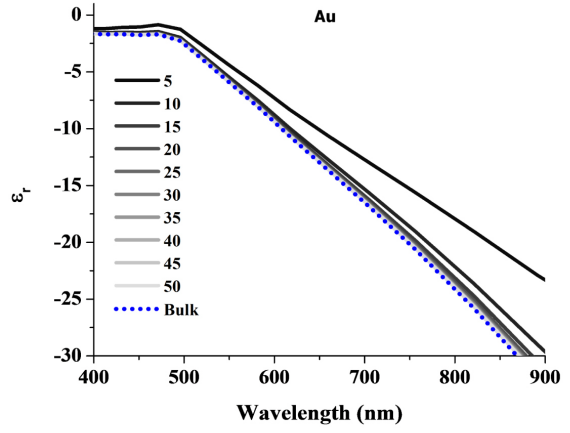

(a)

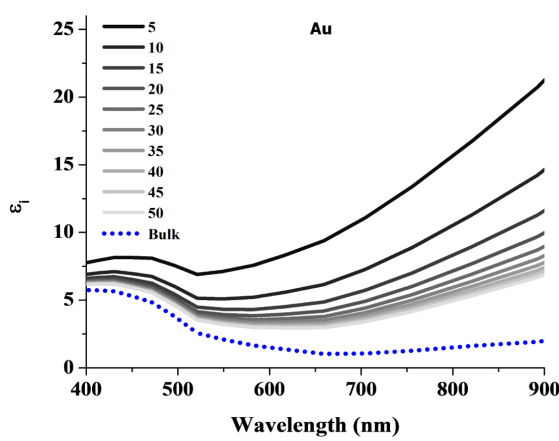

(c)

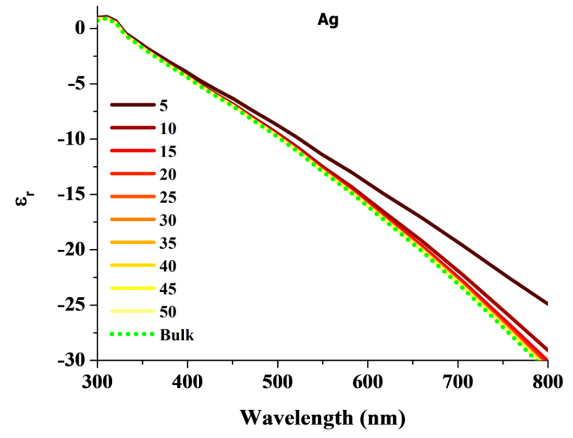

(b)

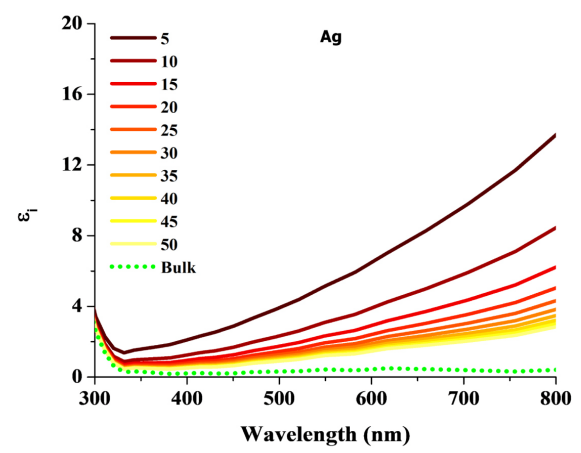

(d)

Figure 1. Complex dielectric function of gold (a) (c) and silver (b) (d) nanospheres with different radius $(5,10,15,20,25,30,35,40,45,50 \mathrm{~nm})$. 
describes energy dissipation or loss in the materials.

Decreasing of the nanoparticle size leads to a lower polarizability of the metal (Figure 1(a) and Figure 1(b)), while increases the magnitude of $\varepsilon_{i}$ (Figure 1(c) and Figure 1(d)). Therefore, higher loss is expected for metallic particle with 5 $\mathrm{nm}$ radius than for $50 \mathrm{~nm}$ radius nanostructures. As $\varepsilon_{r}$ value changes with the nanoparticle size, LSPR peak position and amplitude are also determined by the nanosphere dimension. Figure 2(a) and Figure 2(b) shows the extinction of $\mathrm{Au}$ and Ag nanospheres, respectively, with different sizes (in water). A red-shift of the LSPR extinction is observed by increasing the NP size. Additionally, the spectrum changes are more pronounced for AgNP than for Au nanostructures. Moreover, Ag nanospheres show the presence of quadrupole mode when the radius of sphere exceeds $30 \mathrm{~nm}$, while the most dominant mode for $\mathrm{Au}$ nanospheres is due to dipole resonance.

The E-field profiles around the NP for the dipole mode (gold and silver) and quadrupole mode (silver) can be observed in Figures 2(c)-(e), respectively. The changes in normalized electric field $\left(\left|\mathrm{E} / \mathrm{E}_{0}\right|\right)$ of nanospheres were compared at the LSPR wavelengths. The E-field distribution is expressed by the two lobes for linear polarization and ensures the influence of plasmon dipole. Albeit, four lobes can be examined for quadrupole mode of silver nanosphere of the same radius. The simulations also indicate that the FWHM of the extinction change with respect to the reduction of NP radius. For $5 \mathrm{~nm}$ radius, $\mathrm{Ag}$ and $\mathrm{Au}$ nanospheres the extinction presents a sharp peak, with FWHM $\sim 30.44 \mathrm{~nm}$ and 42.7 $\mathrm{nm}$, respectively. For $50 \mathrm{~nm}$ thick nanoparticle a $155 \mathrm{~nm}$ and $89 \mathrm{~nm}$ FWHM

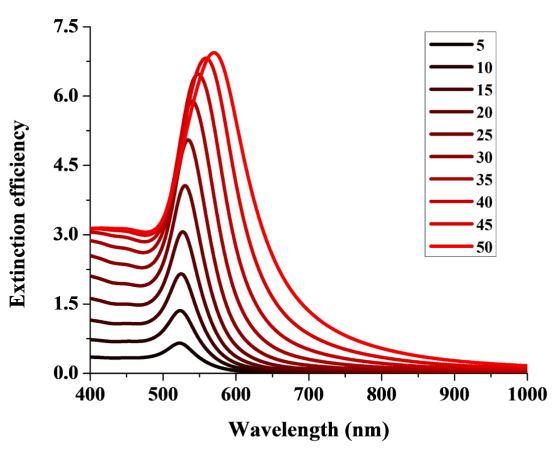

(a)

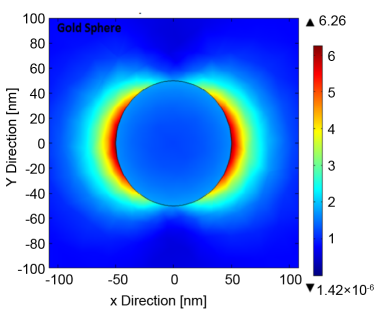

(a)

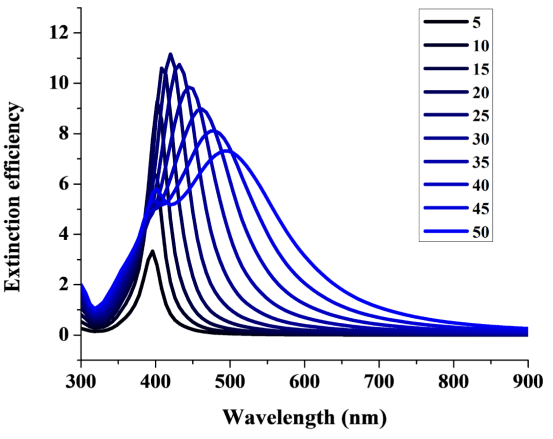

(b)

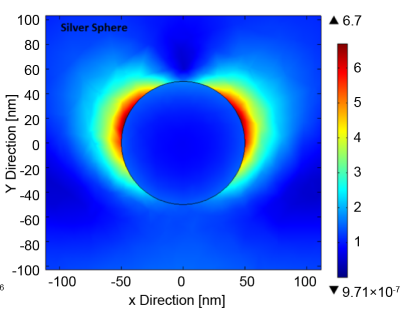

(b)

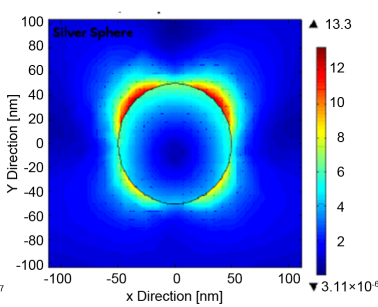

(c)

Figure 2. The LSPR extinction for gold (a) and silver (b) nanospheres in water. E-field profile for gold dipole (c) as well as silver dipole (d) and quadrupole (e) peaks for $50 \mathrm{~nm}$ radius nanosphere. 
were calculated, for the extinction of $\mathrm{Ag}$ and $\mathrm{Au}$ nanoparticles, respectively.

LSPR peak position is determined not only by the real part $\left(\varepsilon_{r}\right)$ of NP dielectric function, but also by the RI of the local medium. On a refractive index based LSPR sensors, bulk sensitivity indicates how LSPR peak position moves with changes on the RI of the local medium.

Figure 3 shows the theoretical and experimental analyses of the LSPR spectral peak position of $25 \mathrm{~nm}$ radius silver and gold nanospheres in several surrounding media, with different RI values. The experimental spectral analysis of the colloidal $\mathrm{Au}$ and $\mathrm{Ag}$ nanospheres were performed in different solvents e.g. water (1.33), acetone (1.36), tetrahydrofuran (1.407), dimethylformamide (1.43), polyethylene glycol-300 (1.465). LSPR peak position is linearly dependent on the refractive indices of surrounding medium as shown in Figure 3(a) for Au and Ag (Figure 3(b)) nanospheres.

For gold nanosphere, with radius of $25 \mathrm{~nm}$, LSPR peak wavelength can shift from $527 \mathrm{~nm}$ to $539 \mathrm{~nm}$ with RI increasing from 1.33 to 1.46, as shown in Figure 3(a). Experimental and theoretical values for the bulk sensitivity of gold spherical nanoparticles were identified as $78 \mathrm{~nm} / \mathrm{RIU}$ and $83 \mathrm{~nm} / \mathrm{RIU}$, respectively. The experimental and theoretical results are in good agreement and indicate the good performance of the simulation method explored. The obtained experimental and theoretical bulk sensitivity values of silver nanospheres were $176 \mathrm{~nm} / \mathrm{RIU}$ and $163 \mathrm{~nm} / \mathrm{RIU}$, respectively. Table 1 presents the bulk sensitivity values of $\mathrm{Au} / \mathrm{Ag}$ nanospheres for radius $25 \mathrm{~nm}$ are in good numerical agreement. The reason in the bulk sensitivity differences of Au versus Ag nanospheres is due to their materials dielectric functions. It is evident (Figure 1) that value of $\varepsilon_{i}$ of silver is smaller than that of gold, in the visible band, resulting higher scattering efficiency and less plasmon damping. This fact makes silver more desirable over gold for sensing applications.

The effects of NP size on the bulk sensitivity and FoM determining nanoparticle sensing platform, can be observed in Figure 4. Ag and Au nanospheres with radii 5 - $50 \mathrm{~nm}$ were analyzed. A theoretical analysis of the bulk sensitivity as

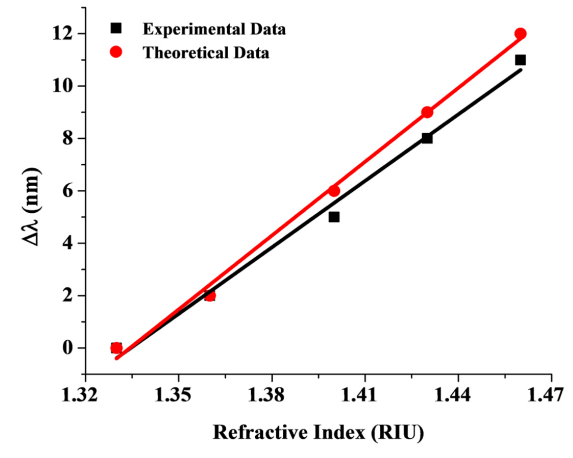

(a)

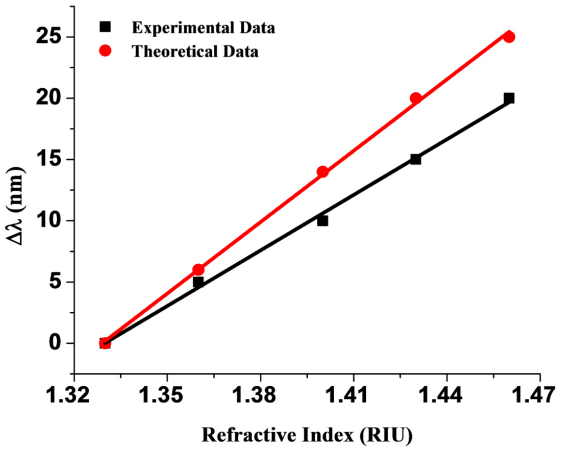

(b)

Figure 3. LSPR peak shift of gold (a) and silver (b) $25 \mathrm{~nm}$ radius nanospheres on changing the refractive index of the surrounding. 


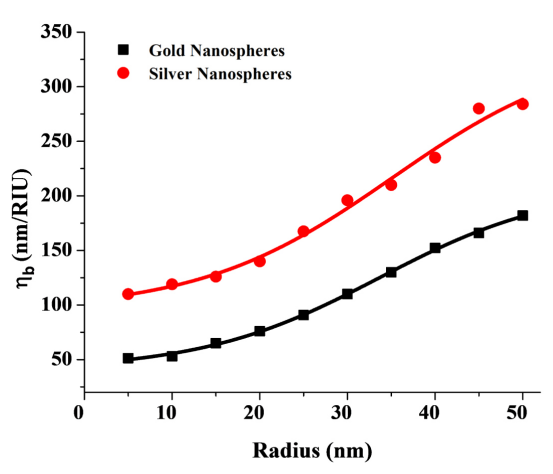

(a)

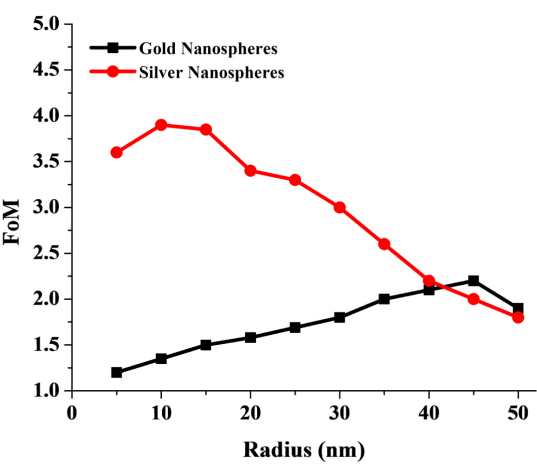

(b)

Figure 4. The bulk sensitivity of gold versus silver nanospheres as a function of particle radius (a) and their respective figure of merit (b).

Table 1. Bulk sensitivity of gold and silver nanospheres ( $25 \mathrm{~nm}$ radius).

\begin{tabular}{ccccc}
\hline Material & Experimental $\eta_{b}(\mathrm{~nm} / \mathrm{RIU})$ & Theoretical & $\eta_{b}$ & $(\mathrm{~nm} / \mathrm{RIU})$ \\
\hline $\mathrm{Au}$ & 78 & 83 \\
$\mathrm{Ag}$ & 176 & 163 \\
\hline
\end{tabular}

function of the NP size (Figure 4(a)) indicates a nonlinear behavior for the $\eta_{B}$ values. As the radius of the nanoparticles enhance, an increase of nanosensor bulk sensitivity from $50-182 \mathrm{~nm} / \mathrm{RIU}$ for gold, and $103-284 \mathrm{~nm} / \mathrm{RIU}$ for silver nanospheres were observed. As NP radius increases, the sensing area also increases, rising the sensitivity of the nanostructured platform. Moreover, for small particle $(r<10 \mathrm{~nm})$ the absorption process manly determines the light-NP interaction.

Scattering phenomenon grows by increasing the particle size $(r>10 \mathrm{~nm})$. For AuNP platform, with $20 \mathrm{~nm}<r<40 \mathrm{~nm}$ bulk sensitivity is highly dependent on the nanostructure size. It can be seen (Figure 4) that bulk sensitivity of nanospheres for Ag is highly influenced on size than to the Au.

The nonlinear behavior of the bulk sensitivity, shown in Figure 4(a), can be empirically described as:

$$
y(r)=A+\left[\frac{B}{1+\mathrm{e}^{\frac{(r-C)}{D}}}\right]
$$

where $A, B, C$ and $D$ are constants and $r$ is the radius of nanospheres.

As the $\mathrm{Ag}$ nanosphere radius increases, the FWHM increase due radiation damping factor [33]. Therefore FoM of Ag nanospheres decrease as the particle size grows, as shown in Figure 4(b). Albeit, the $\varepsilon_{i}$ value of Ag dielectric function is less than that of $\mathrm{Au}$ across visible band, therefore less damping occurs, resulting narrow FWHM (Figure 2(a) and Figure 2(b)) and high values of FoM for silver particles. This is an advantage of $\mathrm{Ag}$ over Au nanospheres in practical biosensing applications. The calculated value of FoM (3.8) of silver nanospheres $(r$ 
$=10 \mathrm{~nm})$ is higher than the reported values of more complex shapes, such as single Au nanorod (1.3) [34], Au nanostar (1.9) [35], Au pyramid (2.2) [8] Ag nanocube (1.6) [36]. The FWHM of gold spectrum is not so affected by increasing the particle size, as compared to Ag nanoplatform. Therefore, the FoM behavior of AuNP is mainly determined by bulk sensitivity changes

On molecular LSPR sensing, the EM field decay length is an important parameter to be considered. As $l_{d}$ value increases the LSPR wavelength shift decreases, due to molecular layer adsorption on a metallic NP surface, as indicated in Campbell's model (equation (1)).

Figure 5(a) shows the EM field decay of an isolated silver nanosphere $(r=5$ $\mathrm{nm})$ in homogeneous surrounding medium $(n=1.33)$. The field decay length can be determined by fitting a single exponential on the field intensity distribution, as proposed by Barbillon et al. [37]. Figure 5(b) demonstrates that EM field decay length increases linearly as the NPs radius grows. For AgNPs with 2.5 $\mathrm{nm}<r<50 \mathrm{~nm}$, the $\mathrm{Ag} l_{d}$ values can be described by a linear fitting as:

$$
l_{d}=G \times r+H
$$

where $G$ (slope) and $H$ are constants (fitting parameters).

Figure 4(b) shows that the EM field decay length of Au/Ag nanospheres can reach few tens of nanometers (at resonance), indicating that LSPR sensors can be highly selective for thinners adsorption layers. While for surface plasmon resonance thin film based sensors the evanescent field decay length is of the order $200 \mathrm{~nm}$ to $300 \mathrm{~nm}$. Campbell's model shows that LSPR peak shift increases by increasing the $\eta_{b}$ values and reducing $l_{d}$. As $\eta_{b}$ and $l_{d}$ are dependent on the nanostructure size, the $\Delta \lambda$ of a LSPR sensor should also be conditioned to the nanosphere radius.

Moreover, Campbell's model also indicates, on increasing the adsorbate layer thickness the LSPR peak shift increases. Figure 6 shows the behavior of LSPR peak shift of the Ag nanosphere sensing platform for different nanostructure size, calculated from Equation (1), (5), and (6). On Figure 6, we assumed that a

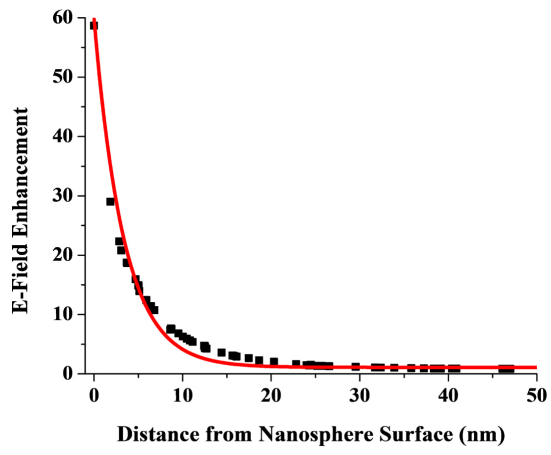

(a)

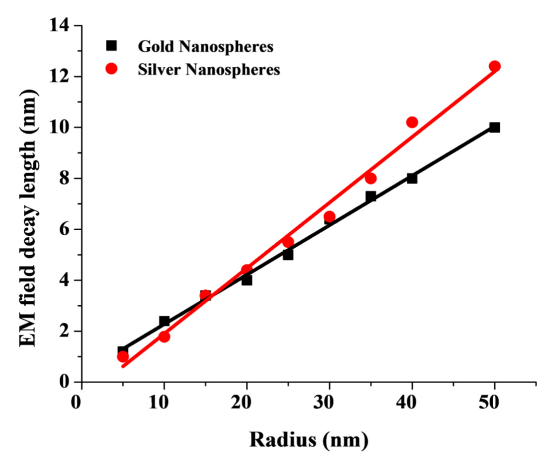

(b)

Figure 5. The electric field decay with respect to distance from the Ag nanosphere $(r=5$ $\mathrm{nm}$ ) surface (a) and $l_{d}$ values for Ag nanosphere with increasing particles radii (b), in homogeneous surrounding medium $(\mathrm{n}=1.33)$. 


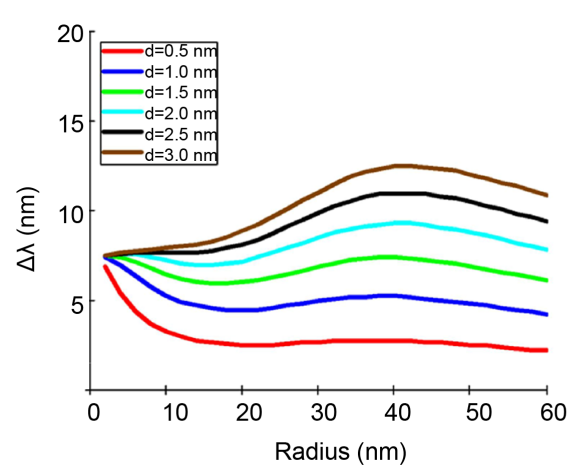

(a)

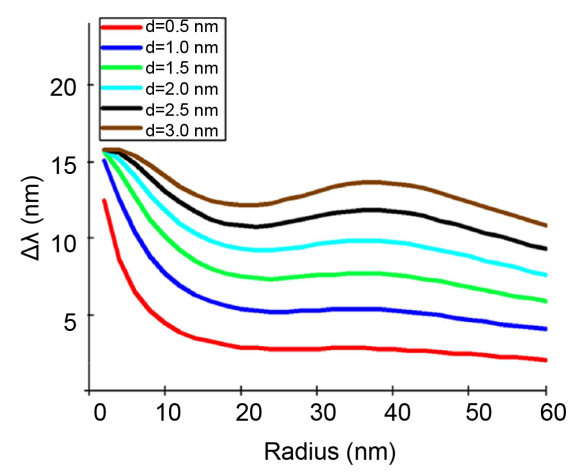

(b)

Figure 6. The LSPR spectral peak shift $(\Delta \lambda)$ as a function of Ag (a) and Au (b) nanoparticles radii by increasing the adsorbate layer thickness, with $n_{\text {ads }}=1.47$.

single monolayer with refractive index equal to 1.47 and different thickness $(0.5$, $1.0,1.5,2.0$, and $3.0 \mathrm{~nm})$, adhered on the NP surface. For small particles $(r \leq 5$ $\mathrm{nm})$, LSPR peak is determined by absorption process, field decay length is limited to few $\mathrm{nm}$ and $\eta_{B}$ values are around $50 \mathrm{~nm} / \mathrm{RIU}$ for gold and $103 \mathrm{~nm} / \mathrm{RIU}$ for silver. Thus high values for $\Delta \lambda$ were observed, about 12 to $17 \mathrm{~nm}$ shift for AgNPs and about 5 to $8 \mathrm{~nm}$ for AuNPs. For lager particles, the $I_{d}$ values increase, increasing the exponential factor on Campbell's model and therefore reducing $\Delta \lambda$ (for $5 \mathrm{~nm} \leq r \leq 20 \mathrm{~nm}$ ). For particles with radius bigger than $25 \mathrm{~nm}$, as NP size increases the bulk sensitivity values considerably increase (Figure 4), and therefore an increase of the LSPR peak shift is observed in Figure 6.

Moreover, Figure 6 shows that $\Delta \lambda$ reduces for particles with radius bigger than $45 \mathrm{~nm}$, due to the fact that bulk sensitivity growth is also reduced (Figure 4). Figure 6 also indicates that, on engineering a LSPR platform, two nanoparticles size range should be considered ( $r$ about $5 \mathrm{~nm}$ or $40 \mathrm{~nm}$ radii). Figure 7 shows the behavior of LSPR peak shift of $5 \mathrm{~nm}$ and $40 \mathrm{~nm}$ metallic (Ag and $\mathrm{Au}$ ) nanospheres for molecular sensing platform, calculated from Equation (1). On Figure 7, it was assumed that a single dielectric shell, with refractive index equal to 1.47 and shell thickness varying from 1 to $10 \mathrm{~nm}$, shielded the NP surface, in water. By the increasing adsorbate shell thickness, the LSPR peak shift increases, for $40 \mathrm{~nm}$ NP radius. Albeit, for $5 \mathrm{~nm}$ NP radius the change on the LSPR spectral shift is not significant $(<2 \mathrm{~nm})$, by growing dielectric shell. Moreover, the slope of the graphics in Figure 7 indicates that for $40 \mathrm{~nm} \mathrm{NP}$ radius, small changes on the dielectric shell thickness can induce significant changes on $\Delta \lambda$. For instance, an increase of the shell thickness from $3 \mathrm{~nm}$ to $6 \mathrm{~nm}$ can induce $12 \mathrm{~nm}$ (for silver) and $\sim 6 \mathrm{~nm}$ (for gold) shift of the LSPR peak wavelength. Many groups have been working on different methodologies to prepare nanoparticles of various structures, leading to high plasmonic fields and tunable plasmon spectrum, from visible to near infrared band. In particular high bulk sensitivity and $F o M$ values were measured for non-spherical structures, like nanorods [34], nanopyramids [8], nanocubes [36] and nanostars [35], particles. The proposed 


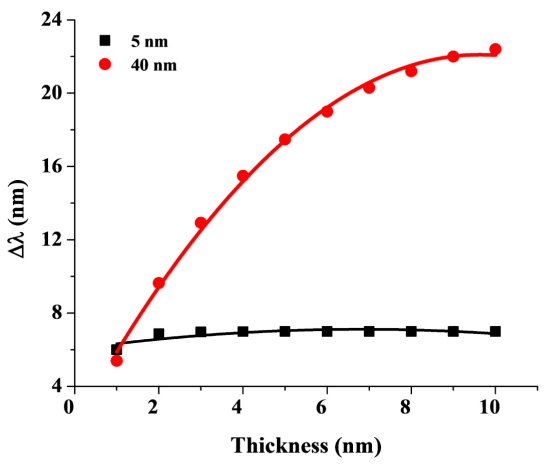

(a)

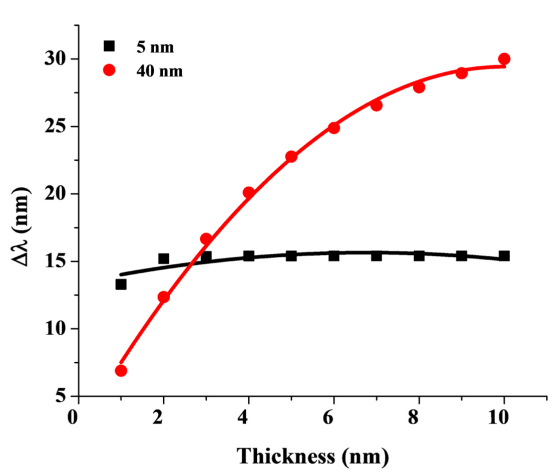

(b)

Figure 7. The study presents the LPSR spectral shift upon the dielectric shell thickness layer for gold nanosphere (a) and silver nanosphere (b).

approached can be extended to engineer the efficiently use of different nanostructures on molecular biosensing.

On medical diagnostic, metallic nanoparticles are base-structures to the establishment of LSPR biosensors. In particular, LSPR label-free immunoassay is launched by functionalizing the NP with an antibody or antigen, by the use of a ligand molecule, as cysteine or cysteamine. Usually, ligand molecules present a thiol functional group that attaches to the metallic nanoparticle surface, and an amine group $\left(-\mathrm{NH}_{2}\right)$, which allows binding to antibodies/antigen carboxyl group. On engineering a high performance LSPR biosensor, the length of the binding molecules (ligand, antibodies and antigen) should be estimated, and a realistic value of the adsorbate shell layer should be used on Campbell's model. For instance, cysteine and cysteamine (ligand molecules) are no longer than $\sim 1$ $\mathrm{nm}$ [19]. Moreover, the refractive index of the adsorbate shell layer should also be appraised. Regardless the different nature of binding molecules, an effective refractive index value of 1.47 RIU can be considered for molecular shell layer on the immunoassay LSPR platform [29].

\section{Conclusions}

Localized Surface Plasmon Resonance phenomenon can drive the development of low-cost and accurate label-free molecular biosensing. The establishment of high performance LSPR biosensor requires the description of sensing parameters (sensibility and $F o M$ ) as function of the nanoparticle structure (size and material).

The obtained theoretical results indicated a nonlinear behavior of the bulk and molecular sensitivity as function of the NP size. Substantial LSPR peak shift due to the adsorption of molecules layer on the NP surface were observed for nanoparticles with $\sim 5 \mathrm{~nm}$ and $\sim 40 \mathrm{~nm}$ radius. Moreover, LSPR peak shift is also determined by the thickness of the adsorbed molecular shell layers. By exploring a $40 \mathrm{~nm}$ radius gold or silver nanospheres, significant LSPR peak shift could be induced by small (few $\mathrm{nm}$ ) thickness change of the adsorbate shell 
layer.

Besides, the engineering approached used in this work provides insights on the LSPR behavior due to adsorption of molecules layer on a NP surface, establishing a new paradigm on engineering LSPR biosensor.

\section{Acknowledgements}

The authors thank the support of INCT Fotônica (CNPq), FACEPE and CAPES.

\section{References}

[1] Mayer, K.M. and Hafner, J.H. (2011) Localized Surface Plasmon Resonance Sensors. Chemical Reviews, 111, 3828-3857. https://doi.org/10.1021/cr100313v

[2] Chen, H., Kou, X., Yang, Z., Ni, W. and Wang, J. (2008) Shape- and Size-Dependent Refractive Index Sensitivity of Gold Nanoparticles. Langmuir, 24, 5233-5237. https://doi.org/10.1021/la800305j

[3] Xu, B.B., Ma, X.Y., Rao, Y.Y., Dong, J. and Qian, W.P. (2011) Plasmonic Biosensors and Nanoprobes Based on Gold Nanoshells. Chinese Science Bulletin, 56, 3234-3241. https://doi.org/10.1007/s11434-011-4670-1

[4] Wang, H., Brandl, D.W., Le, F., Nordlander, P. and Halas, N.J. (2006) Nanorice: A Hybrid Plasmonic Nanostructure. Nano Letters, 6, 827-832.

https://doi.org/10.1021/nl060209w

[5] Skrabalak, S.E., Chen, J., Sun, Y., Lu, X., Au, L., Cobley, C.M. and Xia, Y. (2008) Gold Nanocages: Synthesis, Properties, and Applications. Accounts of Chemical Research, 41, 1587-1595. https://doi.org/10.1021/ar800018v

[6] Fales, A.M. Yuan, H. and Vo-Dinh, T. (2011) Silica-Coated Gold Nanostars for Combined Surface-Enhanced Raman Scattering (SERS) Detection and Singlet-Oxygen Generation: A Potential Nanoplatform for Theranostics. Langmuir, 27, 12186-12190. https://doi.org/10.1021/la202602q

[7] Nusz, G.J., Curry, A.C., Marinakos, S.M., Wax, A. and Chilkoti, A. (2009) Rational Selection of Gold Nanorod Geometry for Label-Free Plasmonic Biosensors. ACS Nano, 3, 795-806. https://doi.org/10.1021/nn8006465

[8] Lee, J., Hasan, W. and Odom, T.W. (2009) Tuning the Thickness and Orientation of Single au Pyramids for Improved Refractive Index Sensitivities. The Journal of Physical Chemistry C, 113, 2205-2207. https://doi.org/10.1021/jp8111155

[9] Song, Y., Nallathamby, P.D., Huang, T., Elsayed-Ali, H.E. and Xu, X.H.N. (2010) Correlation and Characterization of Three-Dimensional Morphologically Dependent Localized Surface Plasmon Resonance Spectra of Single Silver Nanoparticles Using Dark-Field Optical Microscopy and Spectroscopy and Atomic Force Microscopy. The Journal of Physical Chemistry C, 114, 74-81.

https://doi.org/10.1021/jp9083019

[10] Juvé, V. Cardinal, M.F., Lombardi, A., Crut, A., Maioli, P., Pérez-Juste, J., Liz-Marzán, L.M., Del Fatti, N. and Vallée, F. (2013) Size-Dependent Surface Plasmon Resonance Broadening in Nonspherical Nanoparticles: Single Gold Nanorods. Nano Letters, 13, 2234-2240. https://doi.org/10.1021/nl400777y

[11] Jain, P.K. Lee, K.S., El-Sayed, I.H. and El-Sayed, M.A. (2006) Calculated Absorption and Scattering Properties of Gold Nanoparticles of Different Size, Shape, and Composition: Applications in Biological Imaging and Biomedicine. The Journal of Physical Chemistry B, 110, 7238-7248. https://doi.org/10.1021/jp057170o 
[12] Fan, X., Zheng, W. and Singh, D.J. (2014) Light Scattering and Surface Plasmons on Small Spherical Particles. Light. Science \& Applications, 3, 179. https://doi.org/10.1038/lsa.2014.60

[13] Johnson, P.B. and Christy, R.W. (1972) Optical Constants of the Noble Metals. Physical Review B, 6, 4370-4379. https://doi.org/10.1103/PhysRevB.6.4370

[14] Haes, A.J., Chang, L., Klein, W.L. and Van Duyne, R.P. (2005) Detection of a Biomarker for Alzheimer's Disease from Synthetic and Clinical Samples Using a Nanoscale Optical Biosensor. Journal of the American Chemical Society, 127, 2264-2271. https://doi.org/10.1021/ja044087q

[15] Hammond, J.L., Bhalla, N., Rafiee, S.D. and Estrela, P. (2014) Localized Surface Plasmon Resonance as a Biosensing Platform for Developing Countries. Biosensors, 4, 172-188. https://doi.org/10.3390/bios4020172

[16] Takemura, K., Adegoke, O., Takahashi, N., Kato, T., Li, T.C., Kitamoto, N., Tanaka, T., Suzuki, T. and Park, E.Y. (2017) Versatility of a Localized Surface Plasmon Resonance-Based Gold Nanoparticle-Alloyed Quantum Dot Nanobiosensor for Immunofluorescence Detection of Viruses. Biosensors and Bioelectronics, 89, 998-1005. https://doi.org/10.1016/j.bios.2016.10.045

[17] Xu, L., Liu, Y., Chen, Z., Li, W., Liu, Y., Wang, L., Liu, Y., Wu, X., Ji, Y., Zhao, Y., Ma, L., Shao, Y. and Chen, C. (2012) Surface-Engineered Gold Nanorods: Promising DNA Vaccine Adjuvant for HIV-1 Treatment. Nano Letters, 12, 2003-2012. https://doi.org/10.1021/nl300027p

[18] Wang, X., Li, Y., Wang, H., Fu, Q., Peng, J., Wang, Y., Du, J., Zhou, Y. and Zhan, L. (2010) Gold Nanorod-Based Localized Surface Plasmon Resonance Biosensor for Sensitive Detection of Hepatitis B Virus in Buffer, Blood Serum and Plasma. Biosensors and Bioelectronics, 26, 404-410. https://doi.org/10.1016/j.bios.2010.07.121

[19] Camara, A.R., Gouvêa, P.M.P., Dias, A.C.M.S., Braga, A.M.B., Dutra, R.F., de Araujo, R.E. and Carvalho, I.C.S. (2013) Dengue Immunoassay with an LSPR Fiber Optic Sensor. Optics Express, 21, 27023-27031. https://doi.org/10.1364/OE.21.027023

[20] Hong, W., Liang, F., Schaak, D., Loncar, M. and Quan, Q. (2014) Nanoscale Label-Free Bioprobes to Detect Intracellular Proteins in Single Living Cells. Scientific Reports, 4, Article No. 6179. https://doi.org/10.1038/srep06179

[21] Duval Malinsky, M., Kelly, L., Schatz, G.C. and Van Duyne, R.P. (2001) Chain Length Dependence and Sensing Capabilities of the Localized Surface Plasmon Resonance of Silver Nanoparticles Chemically Modified with Alkanethiol Self-Assembled Monolayers. Journal of the American Chemical Society, 123, 1471-1482. https://doi.org/10.1021/ja003312a

[22] Mayer, K.M., Lee, S., Liao, H., Rostro, B.C. and Fuentes, A., (2008) A Label-Free Immunoassay Based Upon Localized Surface Plasmon Resonance of Gold Nanorods. ACS Nano, 2, 687-692. https://doi.org/10.1021/nn7003734

[23] Murray, W.A., Auguié, B. and Barnes, W.L. (2009) Sensitivity of Localized Surface Plasmon Resonances to Bulk and Local Changes in the Optical Environment. The Journal of Physical Chemistry C, 113, 5120-5125. https://doi.org/10.1021/jp810322q

[24] Sherry, L.J., Jin, R., Mirkin, C.A., Schatz, G.C. and Van Duyne, R.P. (2006) Localized Surface Plasmon Resonance Spectroscopy of Single Silver Triangular Nanoprisms. Nano Letters, 6, 2060-2065. https://doi.org/10.1021/nl061286u

[25] Homola, J., Yee, S.S., Gauglitz, G., Sun, Y., Fan, X., Homola, J., Yee, S.S. and Gauglitz, G. (1999) Surface Plasmon Resonance Sensors: Review. Sensors and Actuators B: Chemical, 54, 3-15. https://doi.org/10.1016/S0925-4005(98)00321-9 
[26] Tong, L., Wei, H., Zhang, S. and Xu, H. (2014) Recent Advances in Plasmonic Sensors. Sensors, 14, 7959-7973. https://doi.org/10.3390/s140507959

[27] Sepúlveda, B., Angelomé, P.C., Lechuga, L.M. and Liz-Marzán, L.M. (2009) LSPR-Based Nanobiosensors. Nano Today, 4, 244-251.

https://doi.org/10.1016/j.nantod.2009.04.001

[28] Anker, J.N., Hall, W.P., Lyandres, O., Shah, N.C., Zhao, J. and Van Duyne, R.P. (2008) Biosensing with Plasmonic Nanosensors. Nature Materials, 7, 442-453. https://doi.org/10.1038/nmat2162

[29] Jung, L.S., Campbell, C.T., Chinowsky, T.M., Mar, M.N. and Yee, S.S. (1998) Quantitative Interpretation of the Response of Surface Plasmon Resonance Sensors to Adsorbed Films. Langmuir, 14, 5636-5648. https://doi.org/10.1021/la971228b

[30] Ross, M.B. and Schatz, G.C. (2015) Radiative Effects in Plasmonic Aluminum and Silver Nanospheres and Nanorods. Journal of Physics D: Applied Physics, 48, Article ID: 184004. https://doi.org/10.1088/0022-3727/48/18/184004

[31] Mendoza Herrera, L.J., Arboleda, D.M., Schinca, D.C. and Scaffardi, L.B. (2014) Determination of Plasma Frequency, Damping Constant, and Size Distribution from the Complex Dielectric Function of Noble Metal Nanoparticles. Journal of Applied Physics, 116, Article ID: 233105.

[32] Coronado, E.A. and Schatz, G.C. (2003) Surface Plasmon Broadening for Arbitrary Shape Nanoparticles: A Geometrical Probability Approach. The Journal of Chemical Physics, 119, 3926-3934. https://doi.org/10.1063/1.1587686

[33] Hu, H., Novo, C., Funston, A., Wang, H., Staleva, H., Zou, S., Mulvaney, V., Xiae, Y. and Hartland, G.V. (2008) Dark-Field Microscopy Studies of Single Metal Nanoparticles: Understanding the Factors That Influence the Line Width of the Localized Surface Plasmon Resonance. Journal of Materials Chemistry, 18, 1949-1960. https://doi.org/10.1039/b714759g

[34] Mayer, K.M., Lee, S., Liao, H., Rostro, B.C., Fuentes, A., Scully, P.T., Nehl, C.L. and Hafner, J.H. (2008) A Label-Free Immunoassay Based upon Localized Surface Plasmon Resonance of Gold Nanorods. ACS Nano, 2, 687-692. https://doi.org/10.1021/nn7003734

[35] Nehl, C.L., Liao, H. and Hafner, J.H. (2006) Optical Properties of Star-Shaped Gold Nanoparticles. Nano Letters, 6, 683-688. https://doi.org/10.1021/nl052409y

[36] Sherry, L.J., Chang, S.H., Schatz, G.C., Van Duyne, R.P., Wiley, B.J. and Xia, Y. (2005) Localized Surface Plasmon Resonance Spectroscopy of Single Silver Nanocubes. Nano Letters, 5, 2034-2038. https://doi.org/10.1021/nl0515753

[37] Barbillon, G. (2010) Determination of Evanescent Electric Field Decay Length of Metallic Nanodisks by Using Localized Surface Plasmon Spectroscopy. Journal of Material Sciences and Engineering, 4, 1934-8959.

https://hal-iogs.archives-ouvertes.fr/hal-00626635 